\title{
Enhancement of Brillouin slow-light in optical fibers through optical pulse shaping
}

\author{
Sanghoon Chin and Luc Thévenaz \\ Ecole Polytechnique Fédérale de Lausanne, STI-NAM Station 11, CH-1015 Lausanne, Switzerland \\ sanghoon.chin@epfl.ch, luc.thevenaz@epfl.ch
}

\begin{abstract}
The impact of pulse shape is studied in order to enhance time delaying in Brillouin slow-light. An exponential-like pulse with 14-ns FWHM duration is delayed up to 31-ns in a 27 $\mathrm{MHz}$ wide Brillouin resonance.

(C)2008 Optical Society of America

OCIS codes: (060.4370) Nonlinear optics, fibers; (290.5900) Scattering, stimulated Brillouin; (350.5500) Propagation
\end{abstract}

\section{Introduction}

To date slow-light systems based on stimulated Brillouin scattering (SBS) have proved to be an unmatched and unprecedented flexible tool as a result of their unique spectral tailoring capability. They also show the inherent advantages of a simple experimental configuration, room temperature operation and a large potential bandwidth. Towards the extreme, group velocities as slow as $71,000 \mathrm{~km} / \mathrm{s}$, superluminal and even negative group velocities have been observed through Brillouin slow-light devices [1]. Solutions were proposed to enlarge the bandwidth of Brillouin resonances [2] and to optimize the dispersion characteristics in optical media [3]. All these experiments use the modified Brillouin spectrum by slightly modulating the current applied to the pump laser, so that it can be moderately larger than the pulse spectrum. Although the SBS slow-light element appears to be a very promising timing tool, it has been clear that this scheme needs optimizations to get the maximum benefit of the available bandwidth, since a broader synthesized resonance means a higher pump power and a lower delaying efficiency. We report here an optimization based on the intensity profile of a pulse signal to modify its spectrum for a best match of the Brillouin gain bandwidth. This approach is the first experimental demonstration of a pulse shape optimization on the performance of all-optical delay lines to the best of our knowledge.

\section{Principle}

The relationship between a pulse shape and its optical spectrum can be characterized by its timebandwidth product $\boldsymbol{K}$. This parameter is essentially given by the product of $\Delta v$ and $\Delta t$, defined as the full widths at half maximum (FWHM) of the spectral and temporal distributions, respectively. Table. 1 shows the values of $\boldsymbol{K}$ for various pulse shapes in the case of transform-limited pulses [4]. It turns out that, for a given pulse duration, its spectral width can be substantially modified by properly Table.1. Values of $\boldsymbol{K}$ for various pulse shapes

\begin{tabular}{lll}
\hline Gaussian function & $\varepsilon(\mathrm{t})$ & $\boldsymbol{K}$ \\
\hline Gaussian function & $\exp \left[-\left(\mathrm{t} / \mathrm{t}_{0}\right)^{2} / 2\right]$ & 0.441 \\
Exponential function & $\exp \left[-\left(\mathrm{t} / \mathrm{t}_{0}\right) / 2\right]$ & 0.140 \\
Rectangle & - & 0.892 \\
Lorentzian function & {$\left[1+\left(\mathrm{t} / \mathrm{t}_{0}\right)^{2}\right]^{-1}$} & 0.142 \\
\hline
\end{tabular}
shaping the pulse envelope. Thereby a significant narrowing of the effective spectral width can be obtained. The pulses can therefore be spectrally well confined in the center of the Brillouin resonance where a perfect linear transition in the effective refractive index is observed. As a result, we could achieve a larger pulse delay with a reduced pulse distortion. In a simulation test, we defined three different pulse intensity profiles with identical FWHM duration, showing successively exponential, Gaussian and rectangular temporal distributions. The spectra of the pulses were numerically obtained through a Fourier transform of the pulse waveforms, as shown in Fig.1-(a). It is clearly observed that as anticipated the different pulses show different spectral widths. Then, to demonstrate the validity of the approach, we experimentally generated the three different pulse shapes with identical 14 ns FWHM duration by using an arbitrary waveform generator. The measured pulse spectra show a good agreement with the numerical predictions, especially when considering the spectral narrowing effect. Unavoidable small deviations remain in the spectral width, which are induced by the smoothed edges of the pulses. Among these intensity profiles, the exponential pulse offers the narrowest spectral width of $18 \mathrm{MHz}$, while the Gaussian and rectangular pulses show a measured spectral width of $37 \mathrm{MHz}$ and $62 \mathrm{MHz}$, respectively. 


\section{JMB10.pdf}
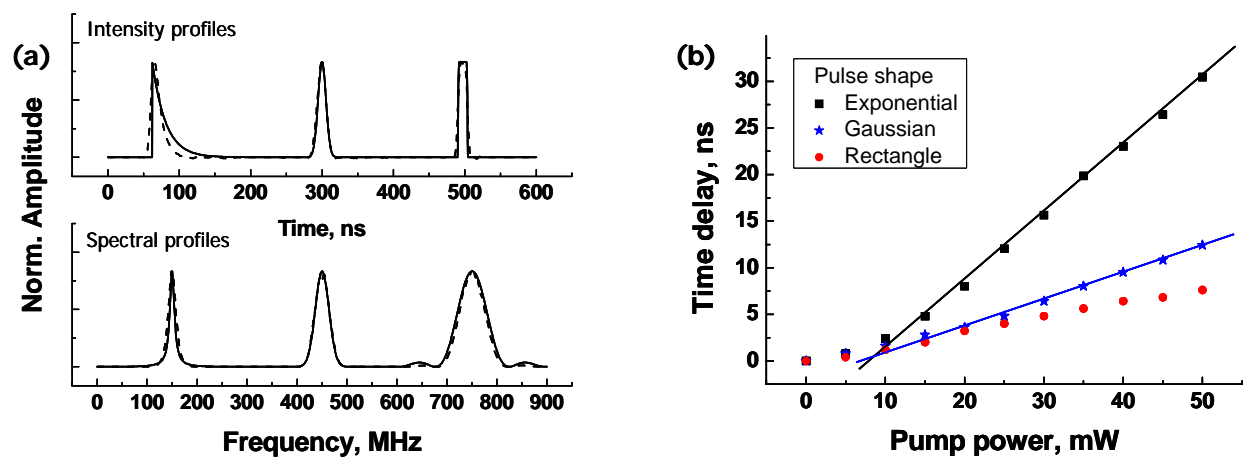

Fig.1: (a) Intensity and spectral profiles of the pulses with exponential, Gaussian and rectangular shapes (left to right). Numerical and experimental results are shown in solid and dashed lines, respectively. (b) Temporal delays of the signal pulses as a function of the pump power.

\section{Experiment and Results}

A CW pump and a pulsed probe signals are generated by modulating the light emitted from a single laser source [5]. A commercial DFB laser diode at $1532 \mathrm{~nm}$ was used as a light source and a 1-km-long conventional single mode fiber was used as a Brillouin gain medium, showing a Brillouin shift of $10.8 \mathrm{GHz}$ and a SBS gain bandwidth of $27 \mathrm{MHz}$. The pump wave was amplified using an Erbium doped fiber amplifier (EDFA) and the output power was controlled by a variable optical attenuator. The signal was frequency-shifted using the sideband technique [5] in an intensity electro-optic modulator to precisely match the Brillouin frequency shift for a maximal gain and then launched into another external modulator to properly shape the pulse signal. This way signal pulse trains with distinct intensity profiles are generated showing identical 14 ns FWHM pulse width.

The temporally delayed pulses after propagation through the fiber are measured as a function of the pump power using a fast detector and displayed on a digital oscilloscope. In all situations the pulses experience more delay while the pump power increases as in typical SBS slow-light systems, but it is clearly observed that the exponentially shaped pulse achieved the largest delay. When rectangular and Gaussian pulses exited the fiber, significant distortion is imposed onto the signal pulses and a reduced time delay is observed, since these two pulses suffer by essence a stronger spectral filtering effect by the here narrower Brillouin resonance. In this experiment we plotted the pulse delays as a function of the pump power for a flat comparison between the time delays obtained in the three different cases, as shown in Fig.1-(b), the effective gain value being substantially different for different pulse shapes. To evaluate the amount of time delay, we determined the peak position of the signal pulse. It is interesting to point out that for a given pump power the achievable time delay is greatly enhanced when the spectral width of the pulse decreases, for fixed input pulse duration. The largest time delay achieved by the exponential pulse is about $30 \mathrm{~ns}$ with a moderate distortion at $50 \mathrm{~mW}$ of pump power and this corresponds to a normalized delay of 2.1. The signal delay also shows a linear dependence on the pump power with a slope efficiency of approximately $0.73 \mathrm{~ns} / \mathrm{mW}$. In the Gaussian case, we also observed that the time delay increases linearly with the pump power and showed a slope efficiency of approximately $0.28 \mathrm{~ns} / \mathrm{mW}$, resulting in a 2.5 times deficiency in the time delaying capability with respect to the exponential pulse.

\section{Conclusions}

We have demonstrated a novel approach to enhance the time delay of SBS slow-light systems through an intelligent shaping of the pulse envelope to optimize the pulse spectral extension. This approach has the advantage to minimize the spectral width for a best matching of the bandwidth of the Brillouin gain resonance. The spectral filtering effect causing a significant signal distortion can be reduced to a large extent. In this optical delaying scheme, a tunable delay of up to $30 \mathrm{~ns}$ equivalent to 2 pulse widths was obtained in the case of exponentially-shaped pulses. The efficiency of the delaying process can thus be improved using that optimized profile by a factor 2.5 compared to a Gaussian pulse.

\section{References}

[1] M. G. Herráez, K. Y. Song and L. Thévenaz, Appl. Phys. Lett., 87, 081113 (2005).

[2] M. G. Herráez, K. Y. Song and L. Thévenaz, Opt. Express, 14, 1395-1400 (2005).

[3] Z. Zhu et al, J. Lightwave Technol, 25, 201-206 (2007)

[4] Zadok, O. Raz, A. Eyal and M. Tur, Photon. Tech. Lett., 19, 462-464 (2007).

[5] Rulliere, Femtosecond Laser Pulses, Springer-Verlag, Berlin Heidelberg, (1998).

[6] M. Niklès, L. Thévenaz and Ph. Robert, J. Lightwave Technol., LT-15, 1842-1851 (1997). 